\title{
Collision of Akhmediev Breathers in Nonlinear Fiber Optics
}

\author{
B. Frisquet, B. Kibler, ${ }^{*}$ and G. Millot \\ Laboratoire Interdisciplinaire Carnot de Bourgogne (ICB), UMR 6303 CNRS-Université de Bourgogne, Dijon, France
}

(Received 1 July 2013; published 19 December 2013)

\begin{abstract}
We report here a novel fiber-based test bed using tailored spectral shaping of an optical-frequency comb to excite the formation of two Akhmediev breathers that collide during propagation. We have found specific initial conditions by controlling the phase and velocity differences between breathers that lead, with certainty, to their efficient collision and the appearance of a giant-amplitude wave. Temporal and spectral characteristics of the collision dynamics are in agreement with the corresponding analytical solution. We anticipate that experimental evidence of breather-collision dynamics is of fundamental importance in the understanding of extreme ocean waves and in other disciplines driven by the continuous nonlinear Schrödinger equation.
\end{abstract}

DOI: 10.1103/PhysRevX.3.041032 Subject Areas: Interdisciplinary Physics, Nonlinear Dynamics, Optics

\section{INTRODUCTION}

Nonlinear coherent phenomena in continuous media have been a key subject of research over the last decades in the framework of the nonlinear Schrödinger equation (NLSE), with applications to plasma physics, fluid dynamics, and nonlinear optics [1]. In particular, much attention has been focused on the common solitary wave structures, known as solitons, and their interactions in almost conservative media. Recently, nonlinear fiber optics has proved its capabilities to demonstrate the existence of solitons (or breathers) on finite background (SFB) solutions to the NLSE [2-4] predicted for more than 25 years. These coherent localized waves evolving on a continuous background exactly describe the dynamical growth of perturbations on a continuous wave related to the nonlinear modulation instability. This includes Akhmediev breathers, Kuznetsov-Ma solitons, and the Peregrine (rational) soliton, which are now considered the simplest models to describe the growth and decay of isolated steep wave events, i.e., rogue waves, in nonlinear dispersive systems [5-9]. But although their first observations were confirmed in different domains of nonlinear science over the past few years, including hydrodynamics [10] and plasma physics [11], no experiment has been performed in order to reveal a synchronized interaction between these first-order periodic solutions of the NLSE. Interaction and collision of common solitons, in contrast, have already been studied in detail [12]; integrable models are known for allowing elastic soliton collisions.

Nonlinear coherent structures are also known to coexist with wave turbulence. Even in the presence of phase

\footnotetext{
*Corresponding author. bertrand.kibler@u-bourgogne.fr

Published by the American Physical Society under the terms of the Creative Commons Attribution 3.0 License. Further distribution of this work must maintain attribution to the author(s) and the published article's title, journal citation, and DOI.
}

randomization inherent to the study of incoherent waves or chaotic states, various numerical works have shown the spontaneous emergence of coherent localized waves (even rational solutions of the NLSE) from a turbulent environment [7,13-15]. Rogue waves are not just an offshoot of breather collisions, but other mechanisms depending on the physical system must be taken into account in the formation of rogue waves, including the statistical approach, when noise is present [16]. The main objective of the present work is to demonstrate the existence of the collision process of Akhmediev breathers in real physical systems, so complete control of all the physical parameters is required in the first phase, which prevents any statistical analysis. It is still of fundamental importance to consider the coherent and deterministic approach to the understanding of rogue-wave phenomena in realistic oceanic conditions $[17,18]$. A recent work has investigated the interaction between waves and ships during extreme ocean conditions using such breather solutions [19]. In this respect, it is also interesting to mention the recent observation of higher-order rational solitons that can explain higher-amplitude rogue waves in deep water [20,21]. These doubly localized waves have been generated in a water-wave tank through nonlinear focusing of initial modulated waves derived from their analytic solutions. However, these higher-order solutions can be interpreted as a nonlinear superposition of two or more rational solutions of first order [7]. As an alternative to describing giant waves, one can consider the collision of first-order periodic solutions of the NLSE theoretically studied in Ref. [7]. Collision between emerging breather structures from waves undergoing modulation instability also appears to be a precursor to the formation of rogue soliton pulses in the process of supercontinuum generation [22]. More generally, collision processes between solitons are known to play an important role in the generation of a supercontinuum [23,24] and the formation of rogue solitons [25] in quasicontinuous-wave pumped-fiber systems. 
Besides a better understanding of extreme events, a first experimental proof of the control of such an interaction between more complex solutions of the NLSE than "ordinary" solitons is an important advance in nonlinear wave propagation for cross-disciplinary research. From a general point of view, the generation of higher-order breathers is of great interest since they offer the possibility of higher energy concentration in space and time, and the generation of waves with giant amplitudes. The large family of breather solutions might become new central objects of nonlinear science thanks to their additional degrees of freedom for energy localization and the spontaneous formation of complex patterns from an initial plane wave, which could be exploited in all domains driven by the NLSE. We report here a novel optical fiber-based test bed using tailored modulated initial conditions of a continuous wave to excite the formation of two Akhmediev breathers $(\mathrm{ABs})$ that collide during propagation. Our experimental work confirms the analytical predictions of breather collision by Akhmediev et al. [7,26]. We have also investigated the synchronization of the collision by controlling the phase and velocity differences between ABs. This work is organized as follows. In Sec. II, we first recall the main properties of single ABs; next, we present the analytical solution (derived by Akhmediev et al.) describing the breather collision and the corresponding numerical predictions in real conditions. In Sec. III, we describe the specific experimental setup implemented here, and we present the experimental results. We also discuss the limitations of our experiment compared to the mathematical ideal. In Sec. VI, we investigate the impact of small variations of initial conditions on the collision phenomenon. In Sec. V, we conclude this work.

\section{ANALYTICAL AND NUMERICAL PREDICTIONS}

The emergence of an isolated steep wave event related to nonlinear modulation instability is explained by the creation of $\mathrm{ABs}$ and, in a more general way, by the family of SFB solutions to the NLSE. The dimensionless selffocusing NLSE is written as follows:

$$
i \frac{\partial \psi}{\partial \xi}+\frac{1}{2} \frac{\partial^{2} \psi}{\partial \tau^{2}}+|\psi|^{2} \psi=0,
$$

where $\psi$ is a wave group or pulse envelope that is a function of $\xi$ (a propagation distance or longitudinal variable) and $\tau$ (a comoving time or transverse variable). The general first-order SFB solution of the NLSE can be written compactly as follows:

$$
\psi_{\mathrm{SFB}}(\xi, \tau)=e^{i \xi}\left[1+\frac{2(1-2 a) \cosh (b \xi)+i b \sinh (b \xi)}{\sqrt{2 a} \cos (\omega \tau)-\cosh (b \xi)}\right] .
$$

Here, the parameter $a$ governs the global physical behavior of the solution in the plane $(\xi, \tau)$ through the function arguments $b=[8 a(1-2 a)]^{1 / 2}$ and $\omega=2(1-2 a)^{1 / 2}$ [4]. (a) Ml gain
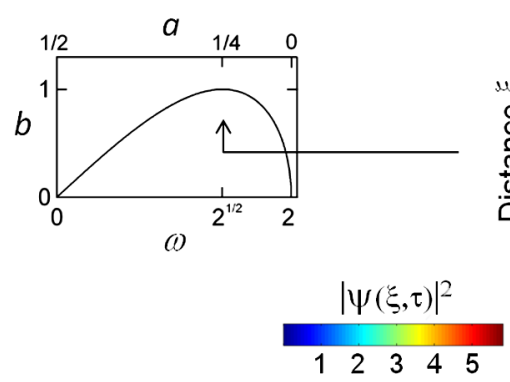

NLSE simulations

(c)

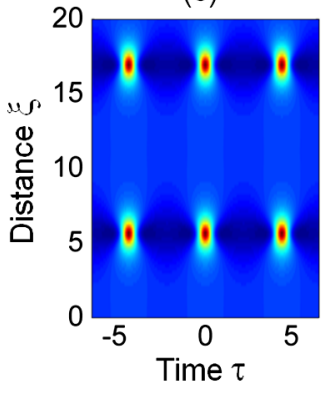

(d)

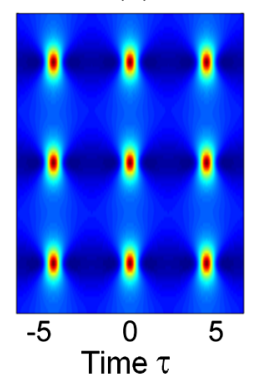

(b) AB theory

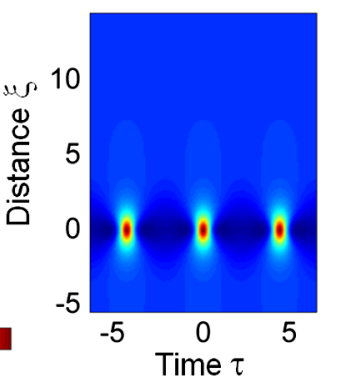

(e)

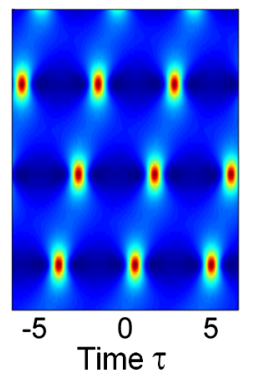

FIG. 1. (a) MI gain related to the general parameter $a$ of $\mathrm{AB}$ theory. (b) False color plot showing the temporal evolution of the exact $\mathrm{AB}$ solution for $a=1 / 4$. Note that only three temporal periods are shown. (c-e) NLSE numerical simulations showing the evolution of distinct (nonideal) initial perturbations on a continuous wave to excite a single $\mathrm{AB}$. (c-d) Cosine perturbations with amplitudes equal to 0.01 and 0.1 , respectively. (e) Exponential perturbation with amplitude equal to 0.1.

In the following, we focus on the particular family of $\mathrm{AB}$ solutions [2], $\psi_{\mathrm{AB}}=\psi_{\mathrm{SFB}}(0<a<1 / 2)$, which describes the nonlinear compression of a modulated continuous wave field into a periodic train of ultrashort pulses with temporal period $T=2 \pi / \omega . \psi_{\mathrm{AB}}$ is valid over the range of modulation frequencies that experience MI gain $(0<\omega<2)$, and the parameter $b>0$ governs the MI growth. The maximum gain condition $b=1$ occurs for $a=1 / 4$, as shown by Fig. 1(a). The corresponding temporal dynamics given by Eq. (2) exhibits only one ideal growth-return cycle in propagation distance [see Fig. 1(b)].

However, certain initial conditions used in practice are known to yield periodic evolution as a function of propagation [2], but even in this case, the first growth-return cycles remain well-described individually by the analytic AB solution. Figures 1(c) and 1(d) illustrate such a biperiodic behavior through the evolution of the following periodic perturbation $\psi_{\mathrm{IN}}=\left[1+\delta_{\bmod } \cos (\omega \tau)\right]$ for two distinct values of amplitude $\delta_{\text {mod }}=0.01$ and 0.1 , respectively. We clearly note that increasing $\delta_{\text {mod }}$ allows us to generate the maximally compressed $\mathrm{AB}$ over a shorter propagation distance. Besides a spectral symmetric seed of the MI process, another simple method is the use of a single-frequency perturbation such as $\psi_{\mathrm{IN}}=$ $\left[1+\delta_{\text {mod }} \exp (i \omega \tau)\right]$, for which longitudinal evolution is 
depicted in Fig. 1(e) with $\delta_{\text {mod }}=0.1$. We again retrieve the previous biperiodic evolution, but the spectral asymmetry of such an input implies that the breather propagates with a certain angle to the line $\tau=0$. Indeed, the clear inclined trajectory comes from the first steps of the initial growth of the perturbation whose spectrum asymmetry induces a distinct mean group velocity of $\mathrm{AB}$ compared to the previous symmetric perturbation. Similar dynamics are also observed in the final decay of the breather cycle. Note that the relative frequency position of the single perturbation to the continuous wave enables the control of the mean group velocity of the AB.

In addition to the simple SFB model, larger extreme waves can also build up when two SFBs collide; the analytic description of the limiting case of $\mathrm{AB}$ collision has been derived in Ref. [7] using Darboux transformations. In that case, the higher-order solution obtained through the nonlinear superposition of first-order solutions depends on the governing parameter of each $\mathrm{AB}(0<$ $\left.a_{1,2}<0.5\right)$. The higher-order solution corresponding to the $\mathrm{AB}$ collision is

$$
\psi_{12}(\xi, \tau)=e^{i \xi}-\frac{4 i \sqrt{2 a_{1}} s_{1} r_{1}^{*}}{\left|r_{1}\right|^{2}+\left|s_{1}\right|^{2}}-\frac{4 i \sqrt{2 a_{2}} s_{12} r_{12}^{*}}{\left|r_{12}\right|^{2}+\left|s_{12}\right|^{2}}
$$

where the parameters $s_{1}, r_{1}, s_{12}$, and $r_{12}$ are also functions of $\xi, \tau, a_{1}$, and $a_{2}$. For the explicit form of Eq. (3), we refer to Ref. [7], where the governing parameter $l$ can be linked to our parameter $a$ through the following relation: $l=$ $i \sqrt{2 a}$. Figure 2 presents the maximal peak power generated by the colliding ABs obtained from Eq. (3) as a function of

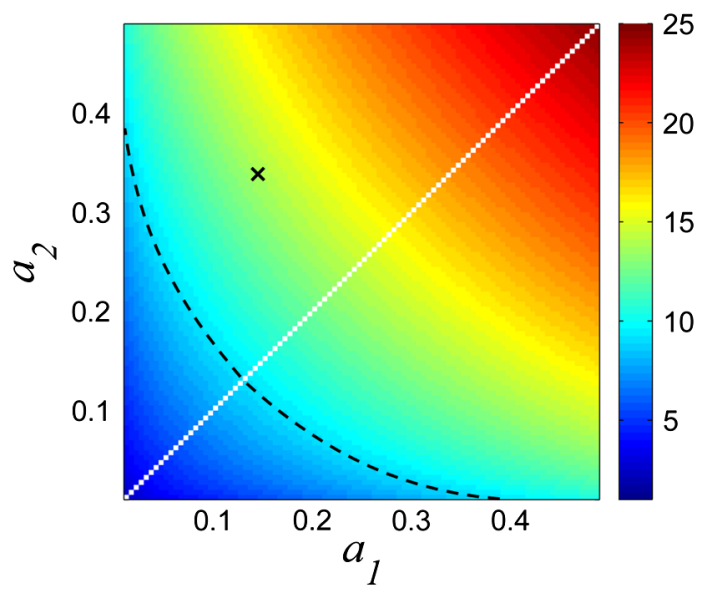

FIG. 2. Maximal peak power $\left|\psi_{12}\right|_{\max }^{2}$ generated by two colliding $\mathrm{ABs}$ as a function of their governing parameter $a$. Note that $1<\left|\psi_{12}\right|_{\max }^{2}<25 .\left|\psi_{12}\right|_{\max }^{2}=1$ corresponds to the background power (i.e., the plane wave). The highest limiting case $\left|\psi_{12}\right|_{\max }^{2}=25$ corresponds to the collision of two Peregrine solitons (i.e., the second-order rational soliton). The dashed black line matches $\left|\psi_{12}\right|_{\max }^{2}=9$, which is the peak power of the Peregrine soliton. The black x corresponds to the physical parameters used in the experiment. their governing parameter. Note that collision can only be studied for two different $\mathrm{ABs}$ (i.e., two distinct values $a_{1}$ and $a_{2}$ ). A more general solution can be found in Ref. [27], which provides a comprehensive understanding of new SFB interactions and their hierarchical nature.

In Fig. 2, the peak power range of the collision is limited by the plane-wave solution (the lowest value) and the second-order rational solution (the highest value). The region above the dashed black line indicates that a large range of collisions can create a giant localized wave with peak power higher than the Peregrine soliton. In the following, our experimental equipment allows us to study the interaction of $\mathrm{ABs}$ in this region of interest. Specifically, the $\mathrm{AB}$ parameters under study are $a_{1}=0.14$ and $a_{2}=$ 0.34 (see Fig. 2). We plot the corresponding solution of Eq. (3) in Fig. 3(a). This particular choice of parameters is driven by our experimental conditions, and it corresponds to commensurate frequencies of $\mathrm{ABs}\left(2 \omega_{1}=3 \omega_{2}\right)$. The resulting wave is periodic in time, but for a better view of the giant wave emerging from the collision, we only highlight one period in Fig. 3. Periodicity of the solution is reported later in the manuscript when the theoretical solution is compared to the experimental results. Nevertheless, Eq. (3) is valid for incommensurate frequencies of $\mathrm{ABs}$; in that case, the nonlinear superposition has one absolute maximum (no periodicity of the collision in time) [7]. Here, we see clearly that breather collision produces a high peak that is doubly localized in the space $(\xi, \tau)$. Note that it is arbitrarily located at the origin. The peak power expected during the collision is about 14 times the initial average power.

More generally, the present work can be linked to the process of higher-order MI known as the simultaneous excitation of multiple instability modes, each mode associated with a corresponding nonlinear breather [28]. The construction of the higher-order solution strongly depends on the synchronization between the centers of the constituent elementary solutions. When the separation is weak, the superposition solution becomes a nonlinear combination of elementary solutions. Here, the synchronization of nonlinear superposition allows colliding $\mathrm{ABs}$ and the generation of a giant wave that strictly differs from simple linear interference. Among previous approaches of experiments on SFB, only single excitation of the MI process has been studied. A single-frequency modulation of the initial continuous (plane) wave is then needed [3,4]. But a small deviation from ideal initial conditions can lead to complex evolution dynamics even for a single SFB [28,29], in particular, when $a>0.375$. Consequently, there is always a tradeoff between the simplicity of the initial modulated wave (inherent to experiments) and the degree of accuracy with which we reach the mathematical ideal. We have used the numerical integration of the NLSE in order to confirm the phenomenon of $\mathrm{AB}$ collision in realistic conditions. First, one can use a superposition of two ideal $A B$ profiles calculated at an arbitrary distance [see Eq. (2)], 
(a) Higher-order solution

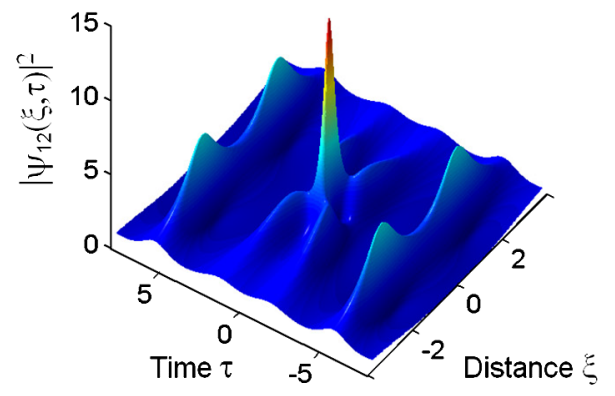

(b) NLSE simulation

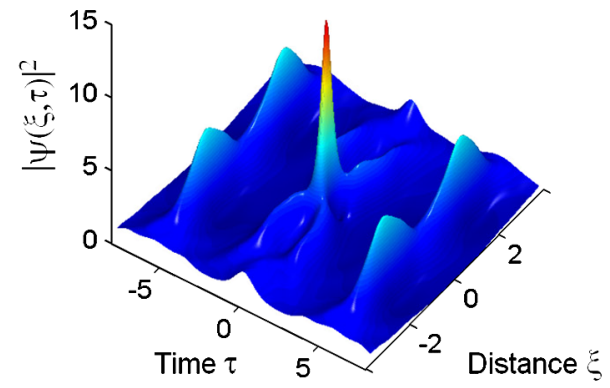

FIG. 3. Collision of two Akhmediev breathers $\left(a_{1}=0.14\right.$ and $\left.a_{2}=0.34\right)$. (a) Corresponding higher-order solution derived from Darboux transformations [see Eq. (3)]. (b) NLSE simulation: Evolution of the superposition of two ideal ABs calculated at arbitrary optimized distances to excite the AB collision. Note that only one period in time is plotted and that initial conditions are arbitrarily injected at $\xi_{0}=-4$ to get the collision at the origin.

$\psi_{\mathrm{IN}}\left(\xi_{0}, \tau\right)=\psi_{a 1}\left(\xi_{1}, \tau\right)+\psi_{a 2}\left(\xi_{2}, \tau\right)$, as input for the simulation. But an efficient collision between both ABs requires, at least, that their maximal intensities coincide in the plane $(\xi, \tau)$ [26]. The appropriate choice of $\xi_{1}$ and $\xi_{2}$ is such that it gives rise to the nonlinear evolution depicted in Fig. 3(b), in excellent agreement with the higher-order solution (here, values of $\left|\xi_{1}\right|$ and $\left|\xi_{2}\right|$ are 1.5 and 1.6, and the input field is normalized so that $\left.\left\langle\left|\psi_{\mathrm{IN}}\left(\xi_{0}, \tau\right)\right|^{2}\right\rangle=1\right)$. Unfortunately, ideal initial conditions involve a complex shaping of the continuous wave [3], which is difficult to achieve in practice.

(a) Single $\mathrm{AB}, a_{1}=0.14$

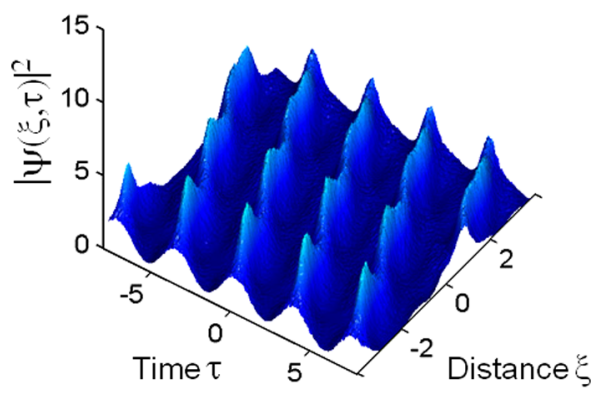

The easiest way is to generate two ABs by seeding the modulation instability process at two distinct frequencies [26]. To this end, we can use a superposition of two complex exponentials for the input field, $\psi_{\mathrm{IN}}=\left[1+\delta_{1} \exp \left(i \omega_{1} \tau\right)+\right.$ $\left.\delta_{2} \exp \left(i \omega_{2} \tau\right)\right]$, where $\omega_{1}, \omega_{2}$ are the frequencies of perturbation, and $\delta_{1}, \delta_{2}$ are small real amplitudes. The initial continuous wave $(\mathrm{CW})$ then contains a bimodulation in the temporal domain. By controlling the initial asymmetry of the two spectral sidebands, we can find specific conditions where the two ABs collide efficiently. Figures 4(a) and 4(b) show the evolution of single breathers obtained with NLSE.

(b) Single $A B, a_{2}=0.34$

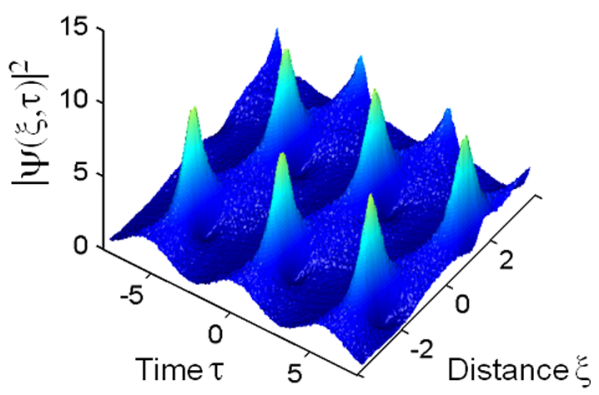

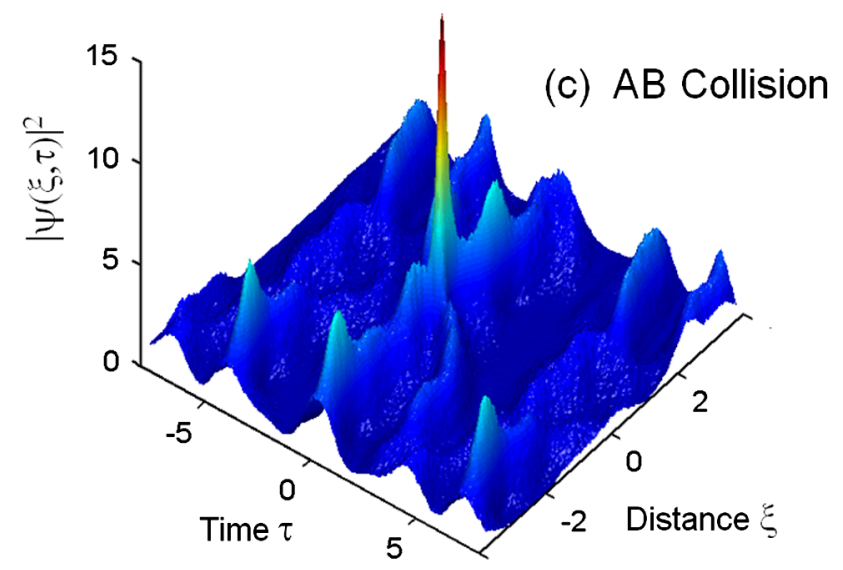

FIG. 4. NLSE simulations: Single breather evolution with nonideal excitation (a) for $a_{1}=0.14$ and (b) for $a_{2}=0.34$. (c) Collision of the two ABs when excited simultaneously by using the superposition of initial conditions of (a) and (b). Note that initial conditions are arbitrarily injected at $\xi_{0}=-4$ to get the collision at the origin for a better comparison with theory (see Fig. 3). 
The adjustments of the sideband amplitudes are the same as those determined for the collision reported below. The spectral asymmetry of such an input implies that a single $\mathrm{AB}$ propagates with a certain angle to the line $\tau=0$; i.e., depending on the relative sideband position, the mean group velocity of the $A B$ is slower or faster than the group velocity of the $\mathrm{CW}$. It is therefore possible to control the group velocity difference between $\mathrm{ABs}$ through a suitable choice of perturbations (with opposite sign of modulation frequency) in order to favor the collision on a short propagation distance.

These simple initial conditions enable the creation of nonideal $\mathrm{ABs}$ so that only the evolution over the first growth-return cycles is described almost accurately by analytical $\mathrm{AB}$ solutions [2]. Note that we present here specific conditions that lead to the collision before the end of the second growth-return cycle of single breather evolutions. Perturbation amplitudes are relatively high in order to keep a short propagation distance (limiting the effect of losses in experiments), $\delta_{1}=0.96$ and $\delta_{2}=0.49$, thus not satisfying the mathematical ideal of $\mathrm{AB}$ theory. Even if large amplitude modulations can excite KuznetsovMa dynamics along the propagation distance [4], we have checked that such initial conditions give rise to spatiotemporal dynamics that are well described by AB theory. A more complete analysis would require the complex use of two-parameter periodic solutions of the NLSE expressed in terms of Jacobi elliptic functions [30].

The numerical simulation corresponding to the initial bimodulated $\mathrm{CW}$ with careful adjustment of $\delta_{1}$ and $\delta_{2}$ shows two colliding $\mathrm{ABs}$ at the shortest distance, here equal to $\left|\xi_{0}\right|=4$, in Fig. 4(c). We observe the emergence of a high-intensity peak at the collision point, in good agreement with the analytical solution shown in Fig. 3(a). The double localization of the giant wave also presents similar widths in both dimensions $\xi$ and $\tau$. Here, the maximal peak power is 14.5 times the initial power. On the other hand, small discrepancies in the form of secondary peaks also arise from the nonideal excitation of ABs. However, it is remarkable to note that the wave evolution obtained with nonideal initial conditions reproduces almost exactly the main giant wave previously predicted.

In conclusion of this section, we have shown from NLSE simulations that $\mathrm{AB}$ collision can be observed even using simple and real excitations of ABs. The experimental investigations of $\mathrm{AB}$ collision are described in the following section.

\section{EXPERIMENTS}

The preliminary NLSE simulations described in Sec. II have allowed us to design experiments where the phenomenon of $\mathrm{AB}$ collision can be tested for the first time. The experimental setup shown in Fig. 5 is mainly based on high-speed telecommunications-grade components. It benefits from a novel fiber-based test bed in comparison to previous studies of single SFB $[3,4,28,29]$. In particular, we have used the spectral shaping of an optical-frequency comb to synthesize tailored modulated initial conditions of the continuous wave. The initial frequency comb is generated by the implementation of a $20-\mathrm{GHz}$ repetition rate pulse source centered at $1550 \mathrm{~nm}$, based on the nonlinear compression of an initial beat signal in a cavityless opticalfiber-based device. More technical details about a similar fiber-based source can be found in Ref. [31]. The spectrum of such a pulse source can be approximated to a series of Dirac delta functions separated by the repetition rate. The width of the comb envelope depends on the nonlinear compression of the initial modulated $\mathrm{CW}$, and it determines the number of sidebands and their decreasing amplitude. Next, a programmable optical filter (wave shaper) provides extremely fine control of the amplitude and phase characteristics across the frequency comb. The high resolution (around $1 \mathrm{GHz}$ ) of this solid-state liquid crystal on silicon (LCoS) system allows us to select or remove individual spectral peaks of the comb. As shown schematically in Fig. 5, a superposition of two complex exponentials for the input field can be created through the spectral shaping of harmonics of the frequency comb. Moreover, the relative amplitude and phase differences of the selected sidebands

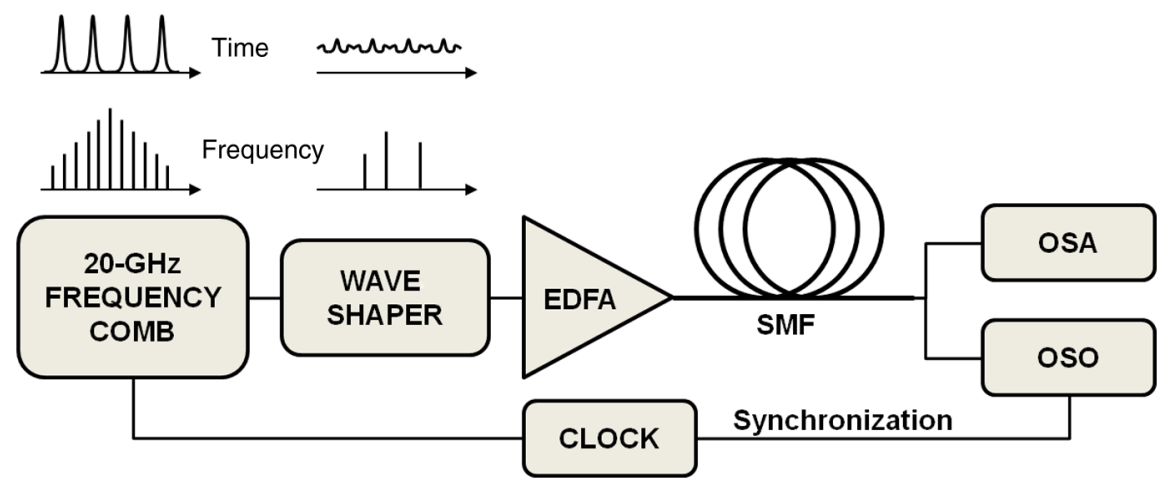

FIG. 5. Experimental setup. 
with the central peak at $1550 \mathrm{~nm}$ (i.e., the continuous wave) are also managed.

In our experiments, an erbium-doped fiber amplifier (EDFA) was used to amplify the average power of the modulated $\mathrm{CW}$ up to $1.1 \mathrm{~W}$. Note that to mitigate the effects of Brillouin scattering, a phase modulator is already included in the 20-GHz-frequency comb system [31]. The optical-wave profile was characterized using an ultrafast optical sampling oscilloscope (OSO) with subpicosecond resolution and a high-dynamic-range optical spectrum analyzer (OSA) with $2.5-\mathrm{GHz}$ resolution. The bimodulated $\mathrm{CW}$ is injected into a standard optical fiber (SMF-28) with group velocity dispersion $\beta_{2}=-20 \mathrm{ps}^{2} \mathrm{~km}^{-1}$ and nonlinearity $\gamma=1.2 \mathrm{~W}^{-1} \mathrm{~km}^{-1}$.

Dimensional distance $z(\mathrm{~m})$ and time $t(\mathrm{~s})$ are related to the previous normalized parameters by $z=\xi L_{\mathrm{NL}}$ and $t=$ $\tau t_{0}$, where the characteristic length and time scale are $L_{\mathrm{NL}}=\left(\gamma P_{0}\right)^{-1}$ and $t_{0}=\left(\left|\beta_{2}\right| L_{\mathrm{NL}}\right)^{\frac{1}{2}}$, respectively. $\omega_{0}$ is the $\mathrm{CW}$ frequency. The modulation frequency $\omega_{\bmod }$ of the $\mathrm{CW}$ is then related to the general parameter $a$ by $2 a=$ $\left[1-\left(\frac{\omega_{\bmod }}{\omega_{c}}\right)^{2}\right]$, with $\omega_{c}^{2}=\frac{4 \gamma P_{0}}{\beta_{2}}$. The dimensional field $A(z, t)\left(\mathrm{W}^{1 / 2}\right)$ is $A=P_{0}^{\frac{1}{2}} \psi, P_{0}$ being the average power of the input wave. Here, the superposition of two complex exponentials for the input field is $A(z=0, t)=$ $\sqrt{P_{0}}\left[1+\delta_{1} \exp \left(i \omega_{\text {mod }, 1} t\right)+\delta_{2} \exp \left(i \omega_{\text {mod }, 2} t\right)\right]$.

We have performed intensive numerical simulations to determine all the parameters of the input wave $A(z=0, t)$ and the collision length. Numerical integration of the generalized nonlinear Schrödinger equation (GNLSE) was used since it is known to provide an accurate description of the propagation of the optical wave $A(z, t)$ in optical fibers [32],

$$
\begin{aligned}
\frac{\partial A}{\partial z}= & -\frac{\alpha}{2} A-i \frac{\beta_{2}}{2} \frac{\partial^{2} A}{\partial t^{2}}+\frac{\beta_{3}}{6} \frac{\partial^{3} A}{\partial t^{3}} \\
& +i \gamma\left[1+\frac{i}{\omega_{0}} \frac{\partial}{\partial t}\right]\left(A(z, t) \int_{-\infty}^{+\infty} R\left(t^{\prime}\right)\left|A\left(z, t-t^{\prime}\right)\right|^{2} d t^{\prime}\right) .
\end{aligned}
$$

The first term on the right-hand side of the equation accounts for the fiber losses (here, $\alpha_{\mathrm{dB}}=4.343 \alpha=$ $\left.0.2 \mathrm{~dB} \mathrm{~km}^{-1}\right)$. The second and third terms describe the second- and third-order dispersion properties $\left(\beta_{3}=\right.$ $\left.0.12 \mathrm{ps}^{3} \mathrm{~km}^{-1}\right)$. The time-derivative term accounts for the self-steepening effects. The GNLSE also describes the instantaneous (Kerr) and delayed (Raman) nonlinear effects described by the response function $R(t)=$ $\left(1-f_{R}\right) \delta(t)+f_{R} h_{R}(t)$. For a complete discussion of the different terms of Eq. (4), we refer the reader to Ref. [32]. Note that GNLSE simulations consider other experimental conditions such as the bandwidth-limited noise of amplified spontaneous emission in the EDFA and a phenomenological one-photon-per-mode background to model quantum noise (the latter was also included in previous NLSE simulations). However, we underline the fact that only the effect of fiber losses significantly affects the previous results obtained with the NLSE model. Indeed, both the spectral bandwidth and peak power of the wave evolving into the fiber are low enough here to avoid the impact of higher-order effects.

We have checked that the phenomenon of $\mathrm{AB}$ collision observed in GNLSE simulations is very similar to that found with the NLSE [see Fig. 4(c)]. In the GNLSE integration, initial conditions are the following: average power $P_{0}=0.83 \mathrm{~W}$, sidebands at $\omega_{\bmod , 1} / 2 \pi=60 \mathrm{GHz}$ and $\omega_{\bmod , 2} / 2 \pi=-40 \mathrm{GHz}$ (i.e., $a_{1}=0.14$ and $a_{2}=$ 0.34 ) with amplitudes $\delta_{1}=0.96$ and $\delta_{2}=0.54$ (note that we slightly increase the initial excitation of the second breather to counteract the effect of fiber losses in comparison to previous NLSE simulations). In Figs. 6(a) and 6(b), we report the experimental observation of the optical-wave profile (red points) injected into the fiber and that obtained at $z=3.8 \mathrm{~km}$ corresponding to the predicted collision distance. These results are compared to GNLSE simulations (blue line), exhibiting very good agreement. We clearly note the presence of a giant wave far above other secondary peaks, as predicted by Fig. 4(c) at the collision point. We retrieve a periodicity in time of the collision phenomenon, which is related to the difference between $\mathrm{AB}$ frequencies (here, $t_{\mathrm{coll}}=3 t_{\mathrm{mod}, 1}=2 t_{\mathrm{mod}, 2}=$ $\left.1 / 20 \mathrm{GHz}^{-1}\right)$. Obviously, when one of the initial sidebands is switched off, we recover the evolution of a single breather [see Figs. 6(c) and 6(d)]. Without an interaction between $\mathrm{ABs}$, the intensity profile of the emerging wave at the fiber output clearly exhibits a lower peak power.

In order to assess the observation of $\mathrm{AB}$ collision in a real physical system, we now compare the experiments to the ideal theory and to the results from NLSE [see Fig. 4(c)] in
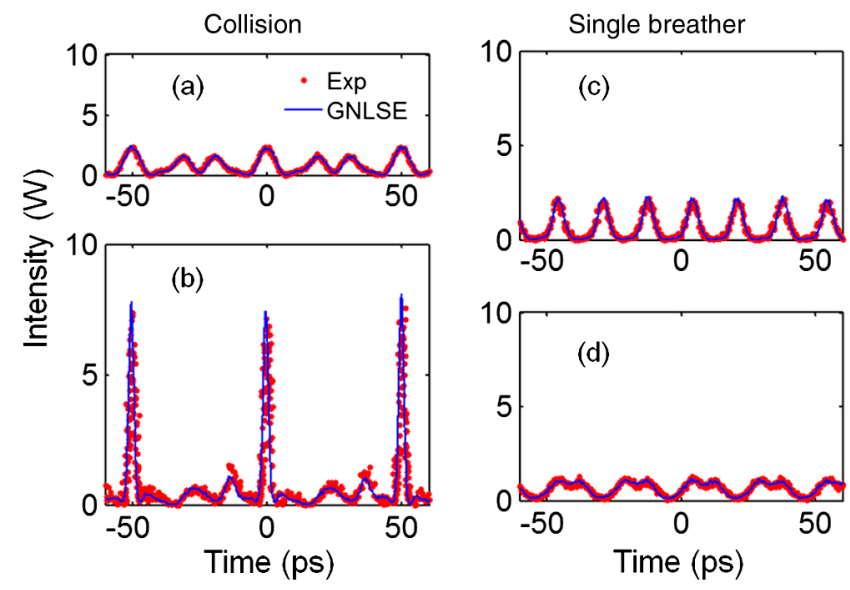

FIG. 6. Temporal intensity profiles obtained before and after propagation into the fiber. (a) Initial condition for two colliding ABs $\left(a_{1}=0.14\right.$ and $\left.a_{2}=0.34\right)$. (b) Giant wave emerging at $3.8 \mathrm{~km}$ (i.e., the collision point). (c-d) Breather profile at $3.8 \mathrm{~km}$ for $a_{1}=0.14$ and $a_{2}=0.34$, respectively, when only one $\mathrm{AB}$ is excited. Experimental profiles (red points) are compared to corresponding GNLSE simulations (blue line). 

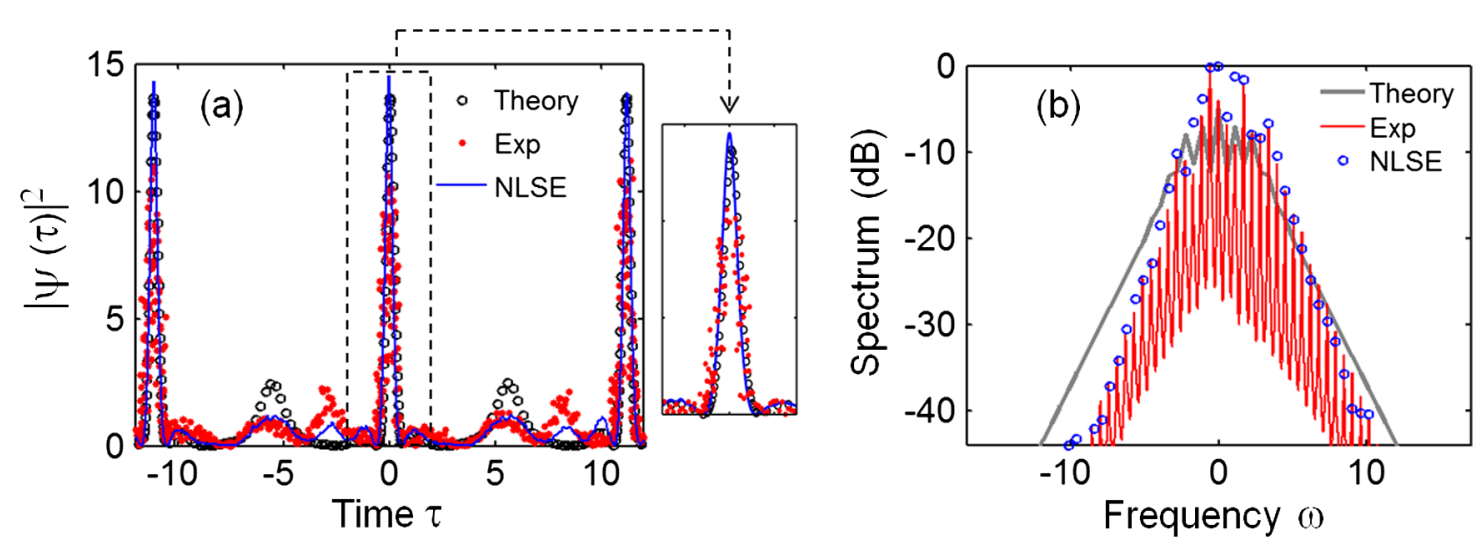

FIG. 7. (a) Experimental collision (red points) profile compared to ideal theory and NLSE simulations with nonideal excitation by using normalized variables. Note that the experimental profile has been normalized here to the average output power. The experimental giant wave reaches almost 11 at maximum, as shown by the zoom on the main peak. (b) Corresponding spectral characteristics from experiment (red) and NLSE simulation (blue circles, shown at peaks only for clarity) and for the ideal higher-order solution (grey).

both the time and frequency domains and using normalized variables. Note that the NLSE prediction of the collision distance $\left(\xi_{0}=4\right)$ is almost satisfied in the experiments where $\xi=\gamma P_{0} z=3.8$. We present in Fig. 7(a) the corresponding normalized wave profiles $|\psi(\tau)|^{2}$ at the collision point. Besides the presence of secondary side lobes attributed to nonideal excitation of $\mathrm{ABs}$, the main discrepancy with the ideal solution from Eq. (3) is the maximal peak power of the giant wave that can be reached. A better agreement would be conceivable if one could excite the collision on a shorter propagation distance. Indeed, removing the loss term in the GNLSE allows us to retrieve the NLSE results. Moreover, we can note that the collision periodicity in time is well predicted by the theory.

Detailed spectral measurements were carried out and shown in Fig. 7(b). Experimental results (red line) are compared to corresponding NLSE simulations (blue circles) in a logarithmic scale, and we see good agreement. The grey line plots the spectrum of the higher-order solution, and it reproduces well the decay of the measured sideband intensities on the high-frequency side. The origin of discrepancies on the low-frequency side is again the nonideal excitation of ABs since the giant wave spectrum created with ideal conditions in NLSE simulations [see Fig. 3(b)] would be indistinguishable from the theoretical one $\left|\widetilde{\psi_{12}}\right|^{2}$. These results are important in showing that the observation of $\mathrm{AB}$ collision is possible even though the ideal higher-order solution exists only in the limit of zero losses and ideal excitation of ABs. In particular, we confirm the creation of a giant localized wave with peak power higher than first-order solutions to the $\operatorname{NLSE}\left(|\psi|_{\max }^{2}>9\right)$.

\section{SYNCHRONIZATION OF AB COLLISION}

In previous sections, the giant peak results from a "synchronized collision" of the two Akhmediev breathers. Indeed, the maximal intensity is obtained when the local maxima of both ABs coincide in the plane $(\xi, \tau)$ [26]. Two characteristics of the excited ABs are known to be crucial, their velocity and phase difference. The control of the velocity difference was performed through the adjustment of both amplitude and frequency of the initial perturbations. In the present section, we study how other variations of the initial conditions impact the collision. First, we simply change the input average power $P_{0}$ of the initial bimodulated wave around the optimal power used before. This change implies that general parameters, $a_{1}$ and $a_{2}$, are slightly modified so that the collision between both $\mathrm{ABs}$ no longer occurs efficiently. The maximal peak power of the temporal profile obtained at the fiber output is reported in Fig. 8(a). The small variation of $a$ values leads to distinct characteristics (distance of compression) of excited ABs and the displacement of their local maxima in the plane $(\xi, \tau)$. Even if ABs can intersect, the analysis of temporal profiles indicates that the combined evolution of $\mathrm{ABs}$ does not necessarily produce a high peak.

Besides the careful choice of input power and properties of initial perturbations described above, the experimental setup allows us to control the spectral phases of exponential functions, $\varphi_{1}$ and $\varphi_{2}$, as follows: $A(z=0, t)=$ $\sqrt{P_{0}}\left[1+\delta_{1} \exp \left(i \omega_{\text {mod, } 1} \tau+i \varphi_{1}\right)+\delta_{2} \exp \left(i \omega_{\text {mod }, 2} \tau+i \varphi_{2}\right)\right]$. Once we fix the phase of one $\mathrm{AB}$, the phase change of the second $\mathrm{AB}$ modifies their relative phase difference $\Delta \varphi=$ $\left|\varphi_{1}-\varphi_{2}\right|$, thus preventing "in-phase" or "synchronized" collision. The corresponding numerical simulations based on the GNLSE are reported in Fig. 8(b); this figure shows a false color plot of the temporal wave profile at the distance of collision, which is analyzed for values of $\varphi_{2}$ in the range $0-2 \pi$, while $\varphi_{1}=0$. We note that the giant wave emerges periodically with $\Delta \varphi$. This $2 \pi / 3$ period is simply related to the initial superposition of two commensurate temporal modulations. The spectral phase shift on the second $\mathrm{AB}$ implies its temporal shift relative to the first $\mathrm{AB}$, so one can recover the same initial condition when $\Delta \varphi=\frac{2 k \pi\left|\omega_{1}-\omega_{2}\right|}{\omega_{1}}$, where $k$ is an integer (here, $2 \omega_{1}=3 \omega_{2}$ ). The experimental 
(a)

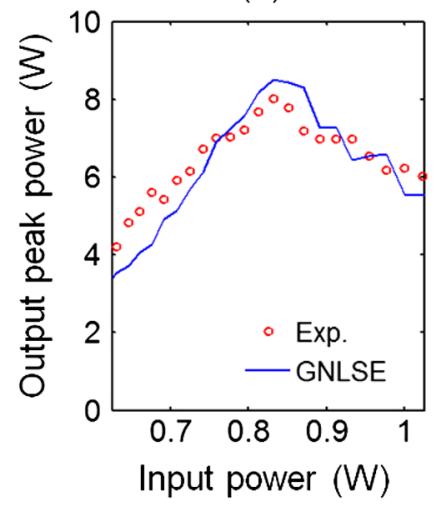

(b)

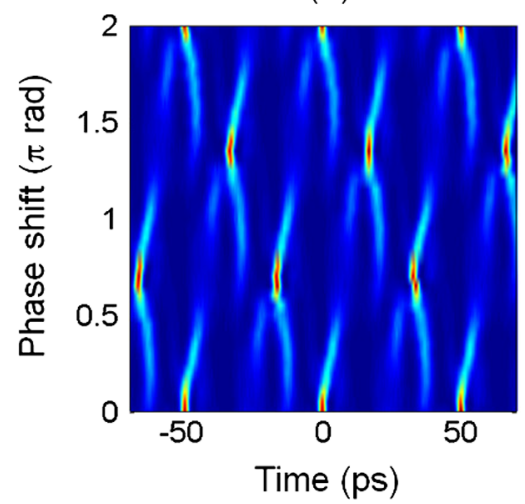

(c)

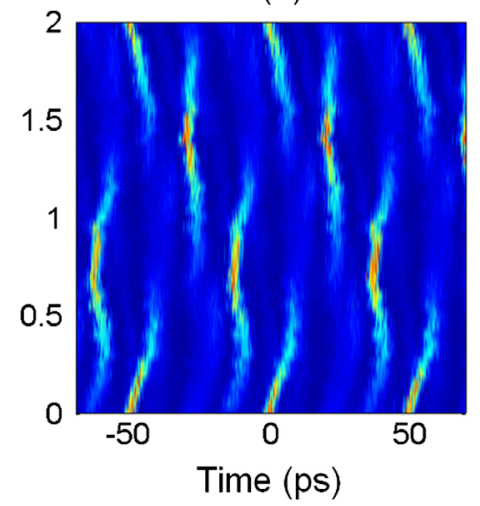

FIG. 8. (a) Highest peak power of the temporal profile recorded at the fiber output as a function of the average input power of the bimodulated wave. (b-c) Evolution of the temporal wave profile as a function of the initial phase difference $\Delta \varphi$ between both ABs. (b) Results from GNLSE simulations and (c) experiments.

results in excellent agreement with simulations are shown in Fig. 8(c). We confirm that the synchronization of the collision between ABs strongly depends on their relative phase difference. Indeed, we are able to suppress the collision event and prevent the emergence of a giant wave by introducing only an initial phase shift between ABs.

\section{CONCLUSION}

We have reported the first experimental observation of collision between Akhmediev breathers in almost conservative media driven by the continuous nonlinear Schrödinger equation. A novel fiber-based test bed using tailored spectral shaping of an optical-frequency comb has been used to excite the formation of two Akhmediev breathers that collide during propagation. Specific initial conditions are required to lead, with certainty, to their efficient collision at an arbitrary point in the plane $(\xi, \tau)$ and the appearance of a giant-amplitude wave whose amplitude is higher than the Peregrine soliton. The qualitative characteristics of the extreme wave are described by the analytical higher-order solution derived from Darboux transformation. Moreover, we have shown that the control of both velocity and phase differences can be used to prevent the emergence of a giant wave. We have also demonstrated that the collision is generated from simple and nonideal initial conditions after a short propagation distance (about 4 nonlinear lengths); this can corroborate the fact that nonlinear coherent structures are known to emerge locally (i.e., a few nonlinear lengths) in a turbulent environment [15]. To conclude, this work opens the way to experimentally investigate interactions between more complex nonlinear coherent structures than "ordinary" solitons. The richer family of multirogue wave solutions of the NLSE is also very attractive for future works. These more general higher-order solutions exhibit multiple maxima, i.e., multiple rogue waves, with a complex spatiotemporal arrangement [27,33-36].

\section{ACKNOWLEDGMENTS}

The authors thank J. Fatome and C. Finot for stimulating discussions. We acknowledge support from the French National Research Agency (ANR-12-BS04-0011 OPTIROC) and the Conseil Régional de Bourgogne through the Photcom PARI grant. This work has been performed in cooperation with the Labex ACTION program (ANR-11-LABX-01-01).

[1] P. L. Christiansen, M. P. Słrensen, and A.C. Scott, Nonlinear Science at the Dawn of the 21st Century, Lecture Notes in Physics (Springer, Berlin, 2000).

[2] J. M. Dudley, G. Genty, F. Dias, B. Kibler, and N. Akhmediev, Modulation Instability, Akhmediev Breathers and Continuous Wave Supercontinuum Generation, Opt. Express 17, 21497 (2009).

[3] B. Kibler, J. Fatome, C. Finot, G. Millot, F. Dias, G. Genty, N. Akhmediev, and J.M. Dudley, The Peregrine Soliton in Nonlinear Fibre Optics, Nat. Phys. 6, 790 (2010).

[4] B. Kibler, J. Fatome, C. Finot, G. Millot, G. Genty, B. Wetzel, N. Akhmediev, F. Dias, and J.M. Dudley, Observation of Kuznetsov-Ma Soliton Dynamics in Optical Fibre, Sci. Rep. 2, 463 (2012).

[5] K. B. Dysthe and K. Trulsen, Note on Breather Type Solutions of the NLS as Models for Freak-Waves, Phys. Scr. T82, 48 (1999).

[6] K.L. Henderson, D. H. Peregrine, and J.W. Dold, Unsteady Water Wave Modulations: Fully Nonlinear Solutions and Comparison with the Nonlinear Schrödinger Equation, Wave Motion 29, 341 (1999).

[7] N. Akhmediev, J.M. Soto-Crespo, and A. Ankiewicz, Extreme Waves that Appear from Nowhere: On the Nature of Rogue Waves, Phys. Lett. A 373, 2137 (2009).

[8] V. I. Shrira and Y. V. Geogjaev, What Make the Peregrine Soliton So Special as a Prototype of Freak Waves? J. Eng. Math. 67, 11 (2010). 
[9] A. R. Osborne, Nonlinear Ocean Waves and the Inverse Scattering Transform (Academic Press, San Diego, 2010).

[10] A. Chabchoub, N. P. Hoffmann, and N. Akhmediev, Rogue Wave Observation in a Water Wave Tank, Phys. Rev. Lett. 106, 204502 (2011).

[11] H. Bailung, S. K. Sharma, and Y. Nakamura, Observation of Peregrine Solitons in a Multicomponent Plasma with Negative Ions, Phys. Rev. Lett. 107, 255005 (2011).

[12] Y.S. Kivshar and G. P. Agrawal, Optical Solitons: From Fibers to Photonic Crystals (Academic Press, San Diego, 2003).

[13] A. Calini and C. M. Schober, Homoclinic Chaos Increases the Likelihood of Rogue Wave Formation, Phys. Lett. A 298, 335 (2002).

[14] A. Ankiewicz, J. M. Soto-Crespo, and N. Akhmediev, Rogue Waves and Rational Solutions of the Hirota Equation, Phys. Rev. E 81, 046602 (2010).

[15] B. Kibler, K. Hammani, C. Michel, C. Finot, and A. Picozzi, Rogue Waves, Rational Solitons and Wave Turbulence Theory, Phys. Lett. A 375, 3149 (2011).

[16] M. Onorato, S. Residori, U. Bortolozzo, A. Montina, and F. T. Arecchi, Rogue Waves and Their Generating Mechanisms in Different Physical Contexts, Phys. Rep. 528, 47 (2013).

[17] M. Onorato, A. R. Osborne, M. Serio, and S. Bertone, Freak Waves in Random Oceanic Sea States, Phys. Rev. Lett. 86, 5831 (2001).

[18] M. Onorato, A. Osborne, M. Serio, L. Cavaleri, C. Brandini, and C.T. Stansberg, Extreme Waves, Modulational Instability and Second Order Theory: Wave Flume Experiments on Irregular Waves, Eur. J. Mech. B, Fluids 25, 586 (2006).

[19] M. Onorato, D. Proment, G. Clauss, and M. Klein, Rogue Waves: From Nonlinear Schrödinger Breather Solutions to Sea-Keeping Test, PLoS One 8, e54629 (2013).

[20] A. Chabchoub, N.P. Hoffmann, M. Onorato, and N. Akhmediev, Super Rogue Waves: Observation of a Higher-Order Breather in Water Waves, Phys. Rev. X 2, 011015 (2012).

[21] A. Chabchoub, N. Hoffmann, M. Onorato, A. Slunyaev, A. Sergeeva, E. Pelinovsky, and N. Akhmediev, Observation of a Hierarchy of up to Fifth-Order Rogue Waves in a Water Tank, Phys. Rev. E 86, 056601 (2012).

[22] G. Genty, C. M. de Sterke, O. Bang, F. Dias, N. Akhmediev, and J. M. Dudley, Collisions and Turbulence in Optical Rogue Wave Formation, Phys. Lett. A 374, 989 (2010).
[23] M. N. Islam, G. Sucha, I. Bar-Joseph, M. Wegener, J. P. Gordon, and D.S. Chemla, Femtosecond Distributed Soliton Spectrum in Fibers, J. Opt. Soc. Am. B 6, 1149 (1989).

[24] M. H. Frosz, O. Bang, and A. Bjarklev, Soliton Collision and Raman Gain Regimes in Continuous-Wave Pumped Supercontinuum Generation, Opt. Express 14, 9391 (2006).

[25] A. Mussot, A. Kudlinski, M. Kolobov, E. Louvergneaux, M. Douay, and M. Taki, Observation of Extreme Temporal Events in CW-Pumped Supercontinuum, Opt. Express 17, 17010 (2009).

[26] N. Akhmediev, J. M. Soto-Crespo, and A. Ankiewicz, How to Excite a Rogue Wave, Phys. Rev. A 80, 043818 (2009).

[27] D. J. Kedziora, A. Ankiewicz, and N. Akhmediev, Second-Order Nonlinear Schrödinger Equation Breather Solutions in the Degenerate and Rogue Wave Limits, Phys. Rev. E 85, 066601 (2012).

[28] M. Erkintalo, K. Hammani, B. Kibler, C. Finot, N. Akhmediev, J. M. Dudley, and G. Genty, Higher Order Modulation Instability in Nonlinear Fiber Optics, Phys. Rev. Lett. 107, 253901 (2011).

[29] K. Hammani, B. Kibler, C. Finot, P. Morin, J. Fatome, J. M. Dudley, and G. Millot, Peregrine Soliton Generation and Breakup in Standard Telecommunications Fiber, Opt. Lett. 36, 112 (2011).

[30] N. Akhmediev and N. Ankiewicz, Solitons, Nonlinear Pulses and Beams (Chapman and Hall, London, 1997).

[31] I. El Mansouri, J. Fatome, C. Finot, M. Lintz, and S. Pitois, All-Fibered High-Quality Stable 20-and 40-GHz. Picoseconds Pulse Generators for 160-Gb/s OTDM Applications, IEEE Photonics Technol. Lett. 23, 1487 (2011).

[32] G. P. Agrawal, Nonlinear Fiber Optics (Academic Press, Oxford, 2013), 5th ed.

[33] P. Dubard, P. Gaillard, C. Klein, and V. B. Matveev, On Multi-Rogue Wave Solutions of the NLS Equation and Position Solutions of the KdV Equation, Eur. Phys. J. Spec. Top. 185, 247 (2010).

[34] P. Dubard and V.B. Matveev, Multi-Rogue Waves Solutions to the Focusing NLS Equations and the KP-I Equation, Nat. Hazards Earth Syst. Sci. 11, 667 (2011).

[35] D. J. Kedziora, A. Ankiewicz, and N. Akhmediev, Circular Rogue Wave Clusters, Phys. Rev. E 84, 056611 (2011).

[36] D. J. Kedziora, A. Ankiewicz, and N. Akhmediev, Classifying the Hierarchy of Nonlinear-SchrödingerEquation Rogue-Wave Solutions, Phys. Rev. E 88, 013207 (2013). 